\title{
Significaciones y preocupaciones de los docentes en torno a la formación de ingenieros en el Tecnológico Nacional de México
}

\author{
Magda Concepción Morales Barrera*| Reinalda Soriano Peña**
}

Este artículo presenta resultados de una investigación que tuvo como propósito comprender las significaciones y preocupaciones de los docentes del Tecnológico Nacional de México en torno a la formación de ingenieros. Desde una perspectiva hermenéutica articulada con herramientas teóricas del análisis político de discurso, se realizó un abordaje metodológico de tipo cualitativo mediante el cual se identificaron y analizaron entendimientos y preocupaciones de los docentes sobre los procesos formativos que desarrollan, mismos que se han publicado en artículos de revistas del propio sistema. Entre los resultados se identificaron significaciones heterogéneas sobre la formación y la ingeniería, sin embargo, hay una preminencia a significar la ingeniería desde una visión operativa-instrumental que se vincula con la reducción del proceso formativo a la capacitación.
Palabras clave

Educación superior tecnológica

Formación de ingenieros

Concepciones del profesor

Práctica educativa

Análisis del discurso

This article presents the results of an investigation aimed towards understanding the teacher's conceptions and concerns regarding the training of engineers at the National Technological Institute of Mexico. In order to do this, we carried out a qualitative methodological approach from a hermeneutical point of view, articulated with theoretical tools of political discourse analysis. We identified and analyzed the teachers' conceptions and concerns, regarding the training processes they develop, which have been published in several articles in specialized journals. Among the results, we found that teachers have a heterogeneous understanding of the meaning of both "training" and "engineering", however, there is a predominance to understand engineering

Keywords

Technological higher education

Engineering training

Teachers' notions

Educational practice

Discourse analysis from an operational-instrumental perspective that tends to result in the reduction of the training process.

Recepción: 17 de marzo de 2020 | Aceptación: 25 de enero de 2021

DOI: https://doi.org/10.22201/iisue.24486167e.2021.174.59811

* Profesora de tiempo completo del Centro Interdisciplinario de Investigación y Docencia en Educación Técnica (CIIDET) del Tecnológico Nacional de México (México). Doctora en Pedagogía. Líneas de investigación: currículo; sujetos y procesos de la educación superior tecnológica. Publicaciones recientes: (2020), "Experiencias de estudiantes de posgrados no escolarizados en México”, en Leticia Pons Bonals (coord.), Experiencia formativa en posgrados a distancia, Puebla, BUAP-BALAM, pp. 137-166; (2019), "La formación de ingenieros en los modelos educativos del Tecnológico Nacional de México: un significante ausente”, Revista de la Educación Superior, vol. 48, núm.192,pp.141-164.CE: mmorales@ciidet.edu.mx

** Profesora de tiempo completo del Centro Interdisciplinario de Investigación y Docencia en Educación Técnica (CIIDET) del Tecnológico Nacional de México (México). Doctora en Pedagogía. Líneas de investigación: políticas educativas; sujetos educativos. Publicaciones recientes: (2020), "La dimensión afectiva y la construcción de subjetividades. La elaboración de la tesis”, Revista Educ@rnos, año 9, núm. 36, pp. 101-115; (2019), “El Tecnológico Nacional de México. Emergencia y procedencia”, Revista de la Educación Superior, vol. 48, núm. 192, pp. 119-139. CE: reynaspea@yahoo.com.mx 


\section{INTRODUCCIÓN}

En este artículo se presentan resultados derivados de la investigación "La formación de ingenieros en el Tecnológico Nacional de México: significaciones y preocupaciones", desarrollada con el objetivo de comprender las significaciones y las preocupaciones respecto a la formación de ingenieros que expresan los docentes del Tecnológico Nacional de México $(\mathrm{TecNM})^{1}$ en distintos foros y medios de difusión y divulgación, con el propósito de responder las siguientes preguntas: ¿qué hacen los docentes en cada institución como práctica de escritura para hacerse escuchar?, ¿cuáles son las significaciones sobre ser ingeniero que están presentes en sus escritos?, y ¿cuáles son sus preocupaciones en torno al proceso formativo de los futuros ingenieros? Por el carácter del objeto de estudio, se abordó desde un enfoque hermenéutico (Heidegger, 1989[1927]), mediante el cual se pretende un acercamiento a los sentidos y significados que los docentes, como actores del proceso formativo, construyen sobre la formación de ingenieros, en un contexto sociohistórico determinado.

La base teórica del trabajo la conforman las nociones de significación y discurso. La noción de significación alude a un proceso intersubjetivo mediante el cual se construyen las concepciones y sentidos con los cuales los sujetos interpretan la realidad social y guían su actuación en los contextos particulares en los que se inscriben. Para entender su complejidad es importante considerar el carácter histórico, contingente e indeterminado que atraviesa al proceso de significación y la función constitutiva que tiene la relación con los otros para que pueda producirse; además, resulta relevante por su función epistémica, al configurar un posicionamiento, entendido como el espacio de enunciación de las prácticas discursivas (De Alba, 2002), desde el cual el sujeto entiende la realidad y actúa en ella con base en ese entendimiento.

En relación con la noción de discurso, éste se entiende como configuración significativa; $y$, en tanto significativa, toda configuración social es discursiva (Laclau, 1993). Así, al hablar de discurso no nos estamos refiriendo a una entidad lingüística, sino a una práctica articulatoria mediante la cual los sujetos construyen sus significaciones y estructuran su posición en un espacio social.

El referente empírico se conformó a partir de la identificación de revistas editadas y publicadas en los Institutos Tecnológicos y Centros que forman parte del TecNM. ${ }^{2}$ Se identificaron 25 revistas (de difusión y divulgación), de las cuales se seleccionaron 11 en las que se ubicaron 78 artículos ${ }^{3}$ donde los docentes expresan preocupaciones en torno al proceso formativo o significaciones sobre la formación de ingenieros en el sistema.

En este artículo se presentan las respuestas encontradas a la interrogante en torno a ¿cuáles son las significaciones sobre ser ingeniero? El escrito está organizado en cuatro apartados: en el primero de ellos se presentan los referentes teórico-conceptuales; en el segundo

\footnotetext{
l Consultar la página web del Tecnológico Nacional de México, en: http://www.tecnm.mx/informacion/sistemanacional-de-educacion-superior-tecnologica (consulta: marzo de 2018).

${ }^{2}$ Cabe aclarar que esta decisión no implica una delimitación lingüística de la noción de discurso, que sería contradictoria con lo planteado en la base conceptual; los motivos de la decisión fueron de carácter técnico-operativo, en función del acceso a la información y a la posibilidad de organizar y difundir la información relativa a los espacios de difusión propios del subsistema.

3 El periodo de publicación de los artículos comprende de 2010 a 2019; las revistas seleccionadas fueron: PulsoTEC (Instituto Tecnológico de La Laguna, Coahuila), Pistas Educativas (Instituto Tecnológico de Celaya, Guanajuato), Cathedra (Instituto Tecnológico de Ciudad Juárez, Chihuahua), Entorno Académico (Instituto Tecnológico Superior de Cajeme, Sonora), Coloquio de Investigación Multidisciplinaria, Innovaitescyt (Instituto Tecnológico Superior de los Cabos, Baja California), Tlahuizcalli (CIIDET, Querétaro), Revista Tecnología Digital (Instituto Tecnológico de Tuxtla Gutiérrez, Chiapas), Revista Tecnológica CEA (Instituto Tecnológico de Roque, Guanajuato), Revista Interdisciplinaria de Ingeniería Sustentable y Desarrollo Social (Instituto Superior de Tantoyuca, Veracruz) e Ingeniantes (Instituto Tecnológico Superior de Misantla, Veracruz).
} 
se describe el método desarrollado; en el tercero se reportan los resultados referidos a las significaciones que los docentes del TecNM han construido sobre la formación de ingenieros y que expresan en los foros de difusión y divulgación institucionales; finalmente se plantean las discusiones y conclusiones, apartado en el que se presenta una síntesis de los resultados y se proponen líneas de debate e interrogación.

\section{SigNIFICACIÓN Y DISCURSO}

Mediante el proceso de significación, los actores del proceso formativo en el $\mathrm{TeCNM}^{4}$ construyen y comparten concepciones y sentidos sobre lo que significa ser ingeniero y hacia dónde debe orientarse su formación; estas significaciones están presentes en sus prácticas educativas y devienen en intereses y preocupaciones que expresan en distintos foros, entre ellos las revistas de difusión y divulgación académicas.

Las significaciones en torno a ser ingenieros - y las implicaciones que éstas tienen sobre la formación de ingenieros en el TecNM- se configuran a partir de elementos del contexto sociohistórico en el que se producen y de las relaciones que establecen con los modos de entender y actuar de los actores; entre estos elementos se pueden mencionar, sin agotar: políticas educativas, modelos educativos y condiciones económicas, sociales, culturales, científicas y tecnológicas. La tarea de quien pretende dar cuenta, desde un marco interpretativo, de los procesos de significación, consistirá en vislumbrar estas influencias y relaciones a partir de las huellas que imprimen en las significaciones que los autores dejan entrever en sus enunciaciones.

Para explicar el concepto de significación que funciona como base teórica de este trabajo, es necesario recurrir a la noción de signo lingüístico planteada por De Saussure (2004[1945]). Uno de los principales aportes de este autor al replanteamiento de la lingüística es su cuestionamiento al reduccionismo que comprende la lengua como nomenclatura, es decir, que establece un vínculo simple entre un nombre y una cosa, para proponer el signo lingüístico como la combinación de dos elementos psíquicos: el concepto (significado) y la imagen acústica ${ }^{5}$ (significante). En la Fig. 1 se muestra el esquema planteado por De Saussure (2004: 92) para representar esta relación.

\section{Figura 1. Representación del signo lingüístico}

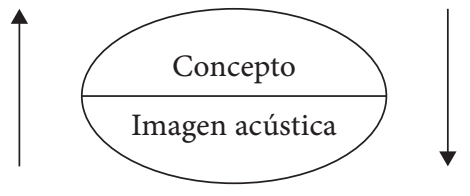

Este entendimiento del signo lingüístico conlleva dos principios: la arbitrariedad del signo y el carácter lineal del significante. El primer principio sostiene que "el lazo que une el significante al significado es arbitrario" (De Saussure, 2004: 93). Este principio es de suma relevancia para entender el carácter sociohistórico de la significación, en tanto sostiene que no hay un vínculo "natural" que dé coherencia al signo lingüístico, sino que éste se estructura mediante convenciones que constituyen reglas producidas colectivamente. No hay, por lo tanto, un valor intrínseco al significante que lo una al significado. Esto no quiere decir, por supuesto, que un individuo o un grupo social pueda cambiar los significados asociados a un significante a su libre arbitrio; eso lo explica el autor ampliamente en

\footnotetext{
4 Aunque los actores del proceso formativo son diversos (estudiantes, docentes, administrativos, directivos, entre otros), en esta investigación nos centramos en los docentes.

5 De Saussure (2004) aclara que la imagen acústica no se refiere al sonido, sino a la huella psíquica de éste.
} 
la condición paradójica de la inmutabilidad del signo lingüístico. Sobre el carácter lineal del significante, De Saussure (2004) señala que éste responde a las condiciones impuestas por la temporalidad, es decir, se estructura en una línea de tiempo, anudándose a otros significantes hasta formar una cadena de sentido.

En estos principios se pueden leer dos supuestos: la construcción histórica, y el carácter relacional del signo, que constituyeron la base de posteriores teorías de la significación y de los movimientos, múltiples y heterogéneos, condensados en lo que se ha denominado giro lingüístico (Lyotard, 1983; Rorty, 1990; Gadamer, 1998). Estos supuestos han reconfigurado distintos campos disciplinarios, como la filosofía, la historia y el psicoanálisis, entre otros, al situar el lenguaje como el eje nodal de sus objetos de estudio.

La noción de significación sobre la que se sostiene este trabajo recupera elementos del psicoanálisis, específicamente de la teoría de Lacan $(1984 ; 2009)$, quien plantea la producción significativa desde dos movimientos: la metáfora y la metonimia; y de la filosofía política, a partir de la propuesta teórica de Laclau $(1993 ; 2001 ; 2006)$ de la cual recuperamos principalmente la lógica de la articulación y la noción de discurso.

Lacan (1984) introduce un giro lingüístico en el psicoanálisis al proponer que el inconsciente se estructura como un lenguaje. Al respecto, invita a leer "bajo la doble rúbrica de la similitud y la contigüidad, lo que sucede en el sujeto delirante alucinatorio" (Lacan, 1984: clase 17). A partir de esta propuesta introduce el interés por la metáfora y la metonimia ${ }^{6}$ como procesos estructurantes del inconsciente, insta a reconocer el lugar nodal del significante y advierte del riesgo de caer en la fascinación del significado. Para explicar la relación entre metonimia y metáfora, Lacan describe dos vínculos internos al significante: el vínculo posicional (metonímico) relativo a la formación de proposiciones mediante las que se instaura el orden de las palabras, y el vínculo de similitud (metafórico), ligado a la posibilidad de la sustitución (una palabra por otra).

Es importante señalar que el vínculo de similitud se construye sobre la base del vínculo posicional. Esto es de particular relevancia para la teoría de la significación propuesta por Lacan (2009), en tanto marca una distancia insalvable entre la estructura del significante y el significado; es decir, lo que está por debajo del significante no es un significado, sino un fluir de significantes enlazados metonímicamente. Por otro lado, la función de la metáfora en la significación es introducir cierta "cesura" al fluir metonímico. De acuerdo con estos planteamientos, la ausencia de significado permite la producción de sentido, que es posible a través de la sustitución mediante la cual el significante ocupa el lugar abierto por esa ausencia, y crea un efecto de sentido que intenta cubrirla.

Las consideraciones de Laclau (1993) respecto a la imposibilidad de plenitud sobre la que se sostienen las estructuras sociales pueden compaginarse con los supuestos lacanianos en torno a la significación; para llevar a cabo este ejercicio analítico, recuperamos de este autor las nociones de articulación y de discurso, las cuales nos permitieron incorporar la dimensión política en nuestras lecturas sobre las significaciones en torno a ser y formar ingenieros en el TecNM. Laclau y Mouffe (1987: 119) definen articulación como:

Toda práctica que establece una relación tal entre elementos que la identidad de éstos resulta modificada como resultado de esta práctica. A la totalidad estructurada resultante de

\footnotetext{
${ }^{6}$ Lacan recupera estas nociones del trabajo sobre las afasias de Jakobson (1956), quien consideró el doble carácter del lenguaje, efectuado por dos operaciones simultáneas: selección y combinación, mediante las que se estructura todo signo lingüístico; a partir de esta consideración elaboró una clasificación de las afasias en trastornos de semejanza y trastornos de combinación. Afirmó entonces que, de los tropos de la figuración retórica, la metonimia es usada por los afásicos con trastornos de semejanza, mientras que los afásicos con trastornos de combinación emplean el lenguaje de modo metafórico.
} 
la práctica articulatoria la llamaremos discurso. Llamaremos momentos a las posiciones diferenciales, en tanto aparecen articuladas en el interior de un discurso. Llamaremos... elemento a toda diferencia que no se articula discursivamente.

La articulación se produce en tanto las identidades que se articulan pueden ser modificadas, esto es, no hay una esencia que las defina, sino que se construyen en la relación con otros; de ahí que la tensión entre interior y exterior, en la que ninguna de las dos esferas es plena, sea su base de producción y, por tanto, "la condición de toda práctica social" (Laclau y Mouffe, 1987: 127). La ausencia de plenitud evoca la falta, y ésta hace posible la tensión entre metáfora y metonimia en el lugar del significado. El carácter constitutivo de esta tensión implica que, si bien la imposibilidad de fijación última (efecto de la metáfora) es el principio de cualquier orden social, también es imposible la dispersión absoluta (libre fluir metonímico).

De acuerdo con lo planteado, el discurso estructurado en la práctica articulatoria no puede configurarse como totalidad porque las relaciones que lo producen son parciales y precarias; las particularidades de los elementos no se logran borrar por completo, penetran la estructura discursiva e impiden su plenitud. Este argumento implica que el discurso, constituido como un intento (fallido) por "detener el flujo de las diferencias, por constituir un centro" (Laclau y Mouffe, 1987: 129), no responde a un principio de necesidad; se configura más bien -histórica y contingentementesobre el horizonte de una plenitud ausente.

Los efectos de estos supuestos, que conforman la base del análisis político de discurso $(\mathrm{APD})^{7}$ sobre el ejercicio analítico que emprendimos, se pueden ordenar en torno a las siguientes consideraciones, las cuales se encuentran estrechamente vinculadas entre sí:
- El carácter histórico y contingente de la significación. No hay un significado absoluto - como una verdad inmanente- desde donde pueda definirse qué es ser ingeniero y tampoco existe un plano de constitución a priori de estas significaciones, ya que se van produciendo a partir de prácticas articulatorias no determinadas.

- La precariedad y parcialidad de las significaciones. Esto implica que las significaciones no son fijas ni permanentes; se movilizan a partir de distintas condiciones (históricas, políticas, económicas, culturales, subjetivas, entre otras).

- La relacionalidad en la construcción de la significación. Aunque la significación sea enunciada por sujetos particulares, está siendo producida en espacios sociales en los que distintos elementos se estructuran en momentos discursivos.

\section{MÉTODO}

A partir de esta investigación se propone la comprensión de un objeto de estudio complejo y poroso, por lo que no se pretende develar ninguna verdad universal o describir sus propiedades de manera que pueda ser plenamente inteligible; lo que se plantea es construir lecturas, si se quiere precarias y parciales, sobre las significaciones que los docentes del TecNM construyen sobre ser ingenieros y las preocupaciones respecto a los procesos formativos que de éstas derivan. De ahí que se haya optado por un enclave metodológico sostenido en la hermenéutica y guiado por los supuestos del APD, lo cual permitió realizar un ejercicio interpretativo capaz de reconocer los contextos discursivos en los que tales significaciones se producen.

La hermenéutica, comprendida desde el APD, se aleja de la aplicación de lógicas y

\footnotetext{
7 Enfoque teórico que toma como eje estos supuestos, trabajado en México por De Alba, Buenfil, entre otros, quienes abordan distintos objetos de estudio, asumiendo el carácter histórico y contingente de la articulación como lógica productora de lo social.
} 
conceptos para constituirse en una práctica articulatoria (Saur, 2005) que pretende "develar verdades estrictamente locales" (Buenfil, 2019: 31), con base en la recuperación de la pregunta por el sentido del ser, articuladora de los intereses ontológicos de Heidegger (1989[1927]) que lo llevaron a reconocer el dasein, situado y construido históricamente. Sobre este reconocimiento se sitúa la empresa hermenéutica, emprendida con la finalidad de interpretar el ser a partir de su estar siendo en el mundo.

De acuerdo con ello, la hermenéutica puede entenderse en tres sentidos: ontológico, en tanto que plantea una analítica de la existencialidad del ser sostenida en su historicidad; epistemológico, relacionado con la elaboración de las condiciones de posibilidad para las investigaciones ontológicas a partir de interrogar sobre el sentido y las estructuras del ser; y metodológico, vinculado al quehacer interpretativo desarrollado por las ciencias comprensivas (Heidegger, 1989).

Si bien nuestras indagaciones se basan en el entendimiento de la hermenéutica a partir de su sentido metodológico, no se pueden soslayar los principios ontológicos y epistemológicos de los cuales se deriva este sentido y que nos llevan a reflexionar sobre la construcción histórica y situada de nuestro objeto de estudio y de las relaciones que establecemos con él. Esto nos permite preguntarnos ¿cómo hacemos jugar la teoría? Esta pregunta sólo puede ser respondida de manera contextual, en tanto los fenómenos sociales de los que pretendemos dar cuenta tienen lugar en contextos sociohistóricos particulares (Buenfil, 2019).

A partir de la precisión anterior, es importante conocer el contexto en el que se sitúa este ejercicio investigativo. Conviene partir describiendo brevemente al TeCNM como un sistema múltiple y heterogéneo, en tanto está conformado por 254 instituciones; ${ }^{8}$ en cuanto al número de instituciones que lo componen y la cantidad de alumnos que atiende, es considerado el sistema de educación superior tecnológica más grande de México. A pesar de ello, hay muy pocas investigaciones orientadas a indagar sobre los procesos formativos de ingenieros que se producen en sus aulas, y las significaciones sobre ser ingenieros que los orientan son escasas (Morales, 2019).

De esa opacidad surgió el interés que ha guiado a este trabajo de investigación, estructurado por la pregunta hermenéutica sobre el sentido de ser ingeniero en el TecNM; la imposibilidad de una respuesta absoluta, debido al carácter histórico y situado del ser, nos exigió centrar la lectura en las voces de los actores y en las condiciones específicas de su producción discursiva.

Con base en ello, se tomó la decisión de construir el referente empírico a partir de las enunciaciones que los autores producen en revistas de difusión y divulgación editadas por algunos de los institutos o centros que pertenecen al TecNM. La dimensión técnica de la investigación, tanto en los momentos de recolección como de análisis de la información, fue de orden cualitativo; para ello se trazó un proceder metodológico organizado por cuatro fases: búsqueda, identificación, organización y análisis.

En la primera fase se hizo un rastreo en la web sobre las revistas editadas por los institutos y centros que conforman el TecNM. La información obtenida se corroboró y completó a partir de una comunicación breve y por vía telefónica al departamento de desarrollo académico de los tecnológicos en los que se ubicaron publicaciones. La intención de este rastreo fue identificar las revistas digitales o impresas publicadas en el sistema, en las que los actores tienen la posibilidad de expresar sus significaciones y preocupaciones en torno a la formación de ingenieros.

\footnotetext{
8126 institutos tecnológicos federales, 122 institutos tecnológicos descentralizados, cuatro centros regionales de optimización y desarrollo de equipo, un Centro Nacional de Investigación y Desarrollo Tecnológico y un Centro Interdisciplinario de Investigación y Docencia en Educación Técnica.
} 
La recuperación de la información en esta primera fase se realizó mediante un instrumento construido exprofeso para clasificar las distintas revistas. Este instrumento se compone de dos secciones: datos de identificación, es decir, información general de la publicación, como nombre, tipo, página web, instituto que la edita, fecha de inicio de publicación, fecha del último número publicado, objetivos y orientación, entre otros aspectos; y condiciones de publicación, centrada en obtener información que dé cuenta de los requisitos para los autores, procesos de arbitraje, indexaciones, posibilidades de acceso, disponibilidad-periodicidad y el tipo de participación que promueve. En esta fase se encontraron 25 revistas.

En la segunda fase se identificaron artículos en los que los autores (específicamente docentes del TecNM) expresan significaciones o preocupaciones en torno a la formación de ingenieros. Se seleccionaron 78 artículos comprendidos en 11 revistas. Estos artículos se desarrollan en torno a temáticas diversas, entre las que destacan: procesos de aprendizaje, didácticas específicas, práctica docente, acreditaciones-certificaciones, organización escolar, cuestiones de género, condiciones medioambientales, reprobación-deserción, retos de la ingeniería y de la formación de ingenieros y usos didácticos de las TIC.

La tercera fase consistió en la organización de la información. Para ello se trabajó con una matriz que permitió extraer, tamizar y organizar las diversas significaciones sobre ser ingeniero construidas por los actores, y las preocupaciones sobre el proceso formativo que de éstas se derivan, categorías eje del trabajo de interpretación realizado. Esta matriz se estructuró en dos niveles: el primer nivel refiere a la categoría significaciones sobre ser ingeniero y se dividió en dos dimensiones: sobre la ingeniería y sobre el proceso formativo. El segundo nivel, ordenado a partir de la categoría preocupaciones en torno al proceso formativo, se estructuró en relación con el punto nodal que articula la preocupación expresada; en torno a ello se plantearon siete elementos, alrededor de los cuales se desarrolló la categoría: el estudiante, modelos educativos y currículo, el docente, gestión y cultura organizacional, relación escuela-mercado laboral, relación escuela-sociedad y temas emergentes.

La cuarta fase consistió en un ejercicio analítico mediante el cual se buscó dar cuenta de la producción de las significaciones sobre ser ingeniero en el TecNM y las preocupaciones que de allí se derivan, a partir de identificar los elementos histórico-contextuales y las relaciones que se entretejen en su producción. Como instrumento para el análisis se utilizaron matrices y mapas conceptuales. Los resultados que se presentan en el siguiente apartado derivan de este ejercicio.

\section{RESUlTADOS}

Paradar respuestaalapreguntasobrelassignificaciones de sery formaringenieros en el TecNM, se identificaron dos significantes nodales: formación e ingeniería; en torno a ellas, los docentes establecen cadenas de significantes y fijan algunos sentidos que configuran las bases del posicionamiento que guía su actuar educativo. Aunque con fines analíticos se trabajaron como elementos distintos, es importante resaltar las articulaciones que establecen entre sí en las configuraciones discursivas resultantes.

\section{Formación}

En torno a la formación se identificaron cadenas metonímicas en las que se producen relaciones entre distintos significantes; hemos organizado estas cadenas en tres significaciones, las cuales se construyen por la operación metafórica que permite fijar un sentido a partir de que un significante se presenta "como si estuviera” en el lugar del significado. Estos significantes son: capacitación, profesionalización y transformación.

Es preciso señalar que las dos primeras significaciones se vinculan al significante com- 
petencias, nodal en los modelos educativos vigentes en el TecNM (DGEST, 2012; TecNM, 2015). Hemos identificado, en torno a este núcleo significante, una pluralidad de sentidos que vale la pena destacar en tanto que permiten poner de relieve la función metonímica en la producción de las significaciones; mediante esta función se articulan elementos heterogéneos que luego son fijados, de manera precaria y parcial, por la operación de la metáfora, misma que, sin embargo, no logra borrar la diferencia que penetra los momentos discursivos.

En este caso, la diferencia se muestra en forma de matices - que entrañan entrecruzamientos y tensiones $-{ }^{9}$ entre distintos marcos de referencia, y que permiten configurar dos entendimientos de las competencias, vinculados: 1) a la adquisición de competencias laborales (sentido articulado con la capacitación) que habilitan a los estudiantes para enfrentar competitivamente las condiciones de los mercados laborales; y 2) al desarrollo de competencias para la formación profesional, en la que se integran conocimientos, habilidades y actitudes (sentido articulado con la profesionalización).

Si bien estas significaciones se han diferenciado a partir de identificar elementos que predominan en cada núcleo significativo, establecen entre sí relaciones múltiples e imbricadas. Al respecto, conviene señalar que, en los modelos educativos vigentes en el TecNM (DGEST, 2012; TecNM, 2015), también se aprecian imbricaciones de elementos heterogéneos en la significación de competencias, ya que se observan tanto sentidos provenientes de la concepción centrada en la formación universitaria, que fue propuesta en el marco europeo a través del proyecto Tuning; como de la concepción estadounidense desarrollada en la década de los setenta para enfrentar la crisis económica de entonces, a partir del entrenamiento laboral (Morales, 2019).

\section{Formación como capacitación}

El entendimiento de los procesos formativos desde su asociación con la capacitación es expresado de manera explícita por algunos docentes que fungen como autores en los artículos seleccionados (Palacios y Dávila, 2013; Romero, 2013; Vázquez y Martínez, 2018, entre otros). En esta significación los docentes asumen un vínculo directo entre los propósitos de su práctica educativa y las necesidades del entorno laboral en el cual se incorporarán los estudiantes.

Esta significación no es ajena a lo planteado en el Modelo Educativo para el Siglo XXI, Formación y Desarrollo de Competencias Profesionales (DGEST, 2012) y en el Modelo de Educación Dual (TecNM, 2015), en los que se asume como premisa formativa atender las necesidades del mercado laboral; en el primer modelo a partir de reducir lo social y profesional, inherente al proceso formativo, a las "necesidades específicas respecto de la demanda laboral" (DGEST, 2012: 33); y en el Modelo de Educación Dual, en el cual esta asociación es más nítida, se apuesta a diseños "flexibles" y súper especializados en los que se integre a la academia y a la empresa para promover que los estudiantes se incorporen "a la vida laboral $\mathrm{y}$ a los procesos productivos del sector empresarial" (TecNM, 2015: 1).

La construcción histórica de esa significación y su relación con los modelos educativos de educación superior, que instan a definir su función a partir de responder a las demandas de los mercados laborales, exigen el reconocimiento de otro vínculo, a saber, el establecido entre las políticas de los organismos internacionales ${ }^{10} \mathrm{y}$ las políticas educativas que han direccionado la educación superior en nuestro país. Conviene destacar la influencia del Banco Mundial (BM), que ha enunciado diversas

${ }^{9}$ La noción de tensión es entendida como una forma de relación específica e irresoluble en la que los elementos que la componen son mutuamente constitutivos y, a la vez, limitantes; Laclau (2006, s/p) plantea que "el momento de tensión se da siempre que hay dos componentes en una relación, que son los dos necesarios, pero sin embargo no pueden ser ajustados automáticamente".

10 Cabe precisar que dichas políticas no son homogéneas ni unidireccionales. 
recomendaciones orientadas a la necesidad de reestructurar los sistemas educativos a partir de reconocer los contextos globales y de competencias económicas (Maldonado, 2000) que exigen capital humano altamente capacitado.

Otro organismo que ha realizado recomendaciones en ese sentido es la Organización para la Cooperación y el Desarrollo Económicos (OCDE), a partir de señalar como prioritaria la relación entre educación superior y empleo. La OCDE recomienda diseños curriculares flexibles que respondan a las demandas productivas, mediante la capacitación para el desarrollo de competencias laborales; respecto a la situación nacional, el organismo afirma que "en México, la educación superior se debe alinear mejor con las necesidades cambiantes de la economía” (OECD, 2019: s/p).

Una asociación interesante en esta forma de entender la formación radica en la relación que se establece entre la función de la educación superior centrada en la capacitación para responder a las demandas de un mercado laboral cada vez más competitivo, y las políticas y mecanismos de acreditación de la calidad, significadas desde nociones traídas de los ámbitos empresariales, como eficacia y eficiencia, que han funcionado como dispositivos tecnopolíticos para reformar los sistemas educativos de la educación superior en distintos países de América Latina, y que han coadyuvado a su alineación con el entorno global neoliberal y su discurso de competitividad (Jiménez, 2011). En este tenor, también se pueden ubicar significaciones que asocian la formación en el TecNM con un procedimiento fabril de manufactura de productos que deben responder a las necesidades del cliente, identificados mediante procedimientos de mercado, por ejemplo, en el caso del texto de Poblano (2012).

Sin pretender agotar todos los hilos de la madeja, se anticipa otra relación - que no se puede obviar - entre el entendimiento de la formación como capacitación y una significación instrumental-operativa de la ingeniería. Sobre este punto se ahondará cuando se planteen las distintas significaciones sobre la ingeniería que se identificaron en los artículos seleccionados.

\section{Formación como profesionalización}

Si bien esta significación se ha estructurado a partir del mismo núcleo significante - el de las competencias- que encuentra sus condiciones de producción en el marco político-económico reseñado, la comprensión se amplía para incorporar elementos que trascienden la habilitación para el desempeño laboral. Entre éstos se ubican:

- Los elementos planteados en el proyecto Tuning (actitudes, conocimientos y habilidades) que conforman las competencias, organizadas en dos tipos: competencias específicas, que corresponden a las que definen el ejercicio profesional de acuerdo a un campo determinado; y competencias genéricas, que aluden al conjunto de competencias que todo profesional debe desarrollar.

- Los aprendizajes planteados por la UNESCO (Delors, 1996) como los pilares sobre los que se deben desarrollar los procesos educativos en las sociedades actuales (aprender a conocer, aprender a ser, aprender a convivir y aprender a ser). - La apuesta por la innovación, entendida en dos dimensiones: sobre la práctica docente, y sobre la formación profesional del ingeniero.

Estos elementos se articulan en distintas formasdeentenderla formación profesionalde los estudiantes y los retos que implican para el docente. En ese tenor, se puede recuperar la voz de Ríos y Soriano (2017), docentes del TecNM, que consideran que las competencias del ingeniero industrial deben desarrollarse desde una visión sistémica que incluya, además de los conocimientos y habilidades propios del ejercicio profesional, elementos axiológicos, 
actitudinales y sociales, entre otros. Este enfoque nos remite a los aprendizajes base de la educación (según Delors, 1996), necesarios para que los egresados puedan responder a las demandas del entorno laboral. Urbina (2011) es otro docente que realiza un planteamiento cercano a esta significación, en tanto que asume que la competencia se conforma a partir de la relación entre distintos elementos, como información, capacidades, hábitos, valores y actitudes, que se demuestran en el desempeño profesional.

Por otra parte, retomamos las voces de Estrada (2012), Romo y Zamora (2013), Romero (2014) y Poblano y Adame (2016), docentes que coinciden en señalar que la formación debe incluir conocimientos, habilidades y actitudes, con lo que dejan ver las huellas discursivas del proyecto Tuning; sin embargo, podemos ubicar algunos matices en sus consideraciones: Estrada (2012) plantea prácticas de construcción, movilización e integración de estos elementos para el ejercicio profesional; Romo y Zamora (2013) y Romero (2014) señalan que la formación debe incluir conocimientos y habilidades para responder competitivamente a las demandas laborales actuales; mientras que Poblano y Adame (2016) se orientan al desarrollo de soluciones eficientes, oportunas y éticas ante los problemas que los egresados enfrentarán en su práctica profesional.

El significante innovación ha cumplido una función central en el entendimiento de la formación profesional en los campos de la ingeniería; sus implicaciones pueden leerse en dos ámbitos: el de las prácticas formativas y el del ejercicio profesional. Respecto al primero, Díaz-Barriga (2006) propone discutir sobre los riesgos de entender la innovación como una superación de lo anterior, sin análisis o reflexión de los procesos, incorporada en el discurso educativo a partir de una perspectiva inmediatista, producida a partir de lógicas políticas e institucionales, pero que no imprimen "nuevos sentidos y significados a su práctica pedagógica” (Díaz Barriga, 2006: 10), en parte porque tampoco se analizan los resultados que han producido.

En tensión (que no contradicción) con este planteamiento, los discursos sobre la innovación se encuentran imbricados con las políticas orientadas a la mejora de la calidad educativa, que apuestan al cambio y la transformación de los procesos y prácticas en las instituciones educativas de educación superior (López, 2017). Aunque esta apuesta conlleva la atención a distintas dimensiones (curriculares, organizacionales, disciplinares, pedagógicas, didácticas, tecnológicas, entre otras), se aprecia una tendencia a asociar la innovación con la incorporación de elementos tecnológicos en las prácticas educativas, especialmente en el ámbito de la didáctica.

En ese tenor, encontramos trabajos de docentes del TecNM que plantean la necesidad de la innovación didáctica (a partir de la incorporación de elementos tecnológicos) para el desarrollo de las competencias en los estudiantes, por ejemplo, García et al. (2015) señalan la importancia del uso de tecnología aplicada a la enseñanza de las matemáticas, y la asocian a la calidad y flexibilidad educativas; mientras que Velasco et al. (2019) proponen la incorporación de la modalidad m-learning, entendida como una metodología innovadora, orientada al aprendizaje, que ha propiciado cambios significativos en la práctica docente y que puede contribuir al desarrollo de competencias específicas en los estudiantes.

En el ámbito del ejercicio profesional de la ingeniería, innovación es un significante que se asocia a las finalidades formativas de la educación tecnológica; de ahí la importancia de revisar los sentidos que se le asignan. Los docentes del TecNM expresan en sus escritos significaciones en torno a la innovación; en ese sentido, recuperamos la voz de Serrano y Castillo (2014), quienes la entienden como una práctica que, anudada a la investigación, permite al ingeniero diseñar y desarrollar actividades encaminadas a la estructuración de proyectos para la solución de problemas propios 
de su campo profesional; Saucedo y Saldívar (2014), en cambio, entienden la innovación como una capacidad asociada a la creatividad que le permite al estudiante de ingeniería emprender y ser competitivo para enfrentar las demandas económicas, laborales y sociales actuales. En otro sentido, Pineda (2014) asume la innovación tecnológica como un eje de desarrollo de la ingeniería a partir del cual puede responder a los desafíos de la economía del conocimiento y fortalecer las relaciones entre academia e industria a partir de la incorporación de "tecnologías de ruptura”. Por su parte, Moguel (2016), sin dejar de reconocer la importancia de la incorporación tecnológica en las prácticas educativas, plantea que el reto de la innovación educativa en la educación tecnológica consiste en responder a los desafíos relacionados con los escenarios cambiantes y la rápida producción del conocimiento.

\section{Formación como experiencia de transformación subjetiva}

Entender la formación como experiencia de transformación subjetiva conlleva una significación amplia del proceso formativo, relacionada profundamente con la configuración identitaria del ingeniero. En este entendimiento, se establece una significación que rompe con la preeminencia atribuida a la formación técnica (tanto en términos de capacitación como de profesionalización) para vislumbrar la necesidad de una base formativa sostenida en la formación del sujeto. Desde esta significación puede establecerse una lectura crítica al planteamiento de la práctica docente que se basa en la trasmisión de conocimientos disciplinarios y, en todo caso, en la habilitación para su aplicación, para entenderla desde un lugar de mediación que posibilita la reconfiguración de las posiciones de sujeto y sus relaciones con el mundo.

En este ámbito de significación resalta la voz de Flores (2012), quien afirma la importancia de resignificar a los alumnos para enten- derlos como sujetos y como ciudadanos que, a partir de la creatividad y la imaginación, sean capaces de transformar la realidad social. En este orden de ideas, el proceso formativo parte de una noción de ciudadano - emancipado- y de un proyecto de nación orientado a reconstruir las tramas sociales con base en el reconocimiento de su historia, para no repetir los errores y fracasos. Repensar la formación desde este marco significativo implica reconocer las múltiples dimensiones que constituyen al ingeniero como sujeto social (epistemológicas, tecnológicas, sociales, culturales, políticas y éticas), las cuales convergen en una trama identitaria que le permite actuar en el mundo.

Cercana a este posicionamiento encontramos la voz de Fuantos (2018), docente del TecNM que sostiene que la formación no se limita a la instrucción, ya que debe incluir la educación como personas; en ese sentido, argumenta que la preparación profesional implica no sólo la capacitación para ejercer de manera competitiva las funciones que atañen al ejercicio profesional del ingeniero, sino que constituye el aprendizaje de una forma de vida y de un modo de relacionarse en la sociedad en el que la formación ética juega un papel fundamental.

\section{Ingeniería}

El otro eslabón de la cadena está conformado por las significaciones sobre la ingeniería, cuestión de relevancia en un sistema que presume de formar al 44 por ciento de los ingenieros de México (TecNM, s/f). Comprender qué se entiende por ingeniería en el TecNM permite dilucidar las implicaciones que esto tiene en el proceso formativo y reconocer las rutas que se están trazando en la educación superior tecnológica, en tanto que estas significaciones sostienen la definición de estructuras curriculares, el diseño de planes y programas de estudio y, por supuesto, las prácticas docentes.

A partir de lo anterior se indagó sobre la conceptualización de la educación superior tecnológica y la formación de ingenieros en 
los modelos que han orientado los procesos educativos en el TecNM, sin embargo, no se encontraron problematizaciones o conceptualizaciones vinculadas con estos significantes. Esta ausencia no significa que haya un vacío de sentido, por el contrario, refleja una significación sostenida en las dicotomías en las que se basa el pensamiento moderno (ciencia-tecnología, teoría-práctica, producciónaplicación del conocimiento), que se asume como plena y universal (Morales, 2019), de ahí que no sea puesta en cuestión. La ingeniería se define, a partir de su función técnica, en el polo aplicativo de esas estructuras dicotómicas, lo que se traduce en mapas curriculares disociados en los que se busca que el ingeniero "adquiera" los conocimientos, con base en la trasmisión, y los “aplique”, sin posibilitar ejercicios reflexivos en torno a tal aplicación.

Más allá de la significación implícita en los modelos educativos, consideramos importante indagar sobre las significaciones que los docentes construyen sobre la ingeniería, en tanto éstas subyacen a las prácticas formativas que desarrollan. La revisión y análisis de los artículos reveló información que permite varias lecturas. La primera de ellas tiene que ver con un incipiente interés sobre el tema: a pesar de que los 78 artículos seleccionados señalan significaciones o preocupaciones en torno al proceso formativo en el TecNM, sólo en 24 de ellos se alude a un entendimiento de la ingeniería como eje rector de tales procesos. En éstos se aprecian huellas de la significación recién esbozada, pero también tensiones que permiten entender la ingeniería como un campo heterogéneo en el que se replantean las relaciones entre ciencia y tecnología producidas en el encuentro de tres racionalidades: científica, técnica y social. A partir de lo anterior, se identificaron cuatro significaciones: instrumental-operativa, politécnica, tecnológica y socio-tecnocientífica.

\section{Significación instrumental-operativa}

Esta significación es la de mayor prevalencia, pues está presente en 12 de los 24 artículos en que se identificó algún sentido de la ingeniería; sin embargo, se puede inferir que la falta de posicionamiento explícito en torno a esta disciplina, aunada al entendimiento de la formación como un proceso de capacitación (como entrenamiento) y a preocupaciones inmediatistas sobre el proceso formativo, centradas en la instrumentalización de la didáctica o en la trasmisión de algún contenido específico, son signos que evidencian una significación instrumental-operativa de la ingeniería.

Desde esta significación, la ingeniería se concibe como una actividad operativa, en la que los conocimientos científicos se "instrumentalizan" para dar solución a problemas técnicos concretos. La relación entre ciencia y tecnología es lineal y simplificada, de ahí que no haya mayor preocupación por entender las estructuras y los conceptos que configuran las bases científicas, en tanto lo más importante, desde esta perspectiva, es la memorización de contenidos aislados y su aplicación mecanicista; esto genera una tendencia a la homogeneización reduccionista de los contenidos, resultante de su instrumentalización y de la invisibilización de las relaciones conceptuales y curriculares que se establecen.

Por otro lado, se encontró una relación fuerte entre esta significación y los planteamientos del Modelo Dual (TecNM, 2015), centrado en la habilitación técnica de los estudiantes para que puedan responder a los requerimientos particulares de una empresa determinada. La apuesta formativa resultante de esta visión radica en la superespecialización, condicionada por los entornos laborales en los que el ingeniero participará como operario, y se centra en el desempeño, el cual se apareja al "saber hacer" y subordina otros saberes necesarios. 
De los artículos revisados, sólo en cuatro se aprecian sentidos que podrían apuntar a una significación politécnica de la ingeniería; esto resalta, sobre todo, si se considera que el surgimiento de la educación superior tecnológica en el país estuvo influido por el modelo politécnico francés (Weiss y Bernal, 2013), que considera el conocimiento científico (principalmente de las matemáticas y la física) como la base formativa del ingeniero, mientras que asume la técnica como resultante de la aplicación de este conocimiento. Cabe destacar que aún se aprecian huellas de este modelo en las estructuras curriculares y académicas del TecNM.

Es importante precisar que la relación ciencia-tecnología sobre la que se sostiene esta significación es distinta respecto a lo planteado en la significación instrumental-operativa de la ingeniería; aunque en ambas se parte de una relación lineal entre ciencia y tecnología, donde la segunda resulta de la aplicación de la primera, en la visión instrumental operativa los conocimientos científicos se reducen a herramientas (de ahí su simplificación conceptual y el aislamiento curricular), mientras que en la significación politécnica configuran la base a partir de la cual el ingeniero puede entender y solucionar los problemas que enfrenta en su ejercicio profesional.

En esta concepción se apuntalan relaciones multidisciplinarias articuladas por la estructura denominada ciencias básicas, a partir de las cuales se pretende que el ingeniero en formación adquiera los conocimientos necesarios para formar un pensamiento lógico que le permita comprender los fenómenos que enfrentará en su práctica profesional y diseñar, con base en ellos, las soluciones pertinentes. En esa pretensión se aprecia el reconocimiento de la complejidad de las relaciones conceptuales que configuran la base científica, por lo que su enseñanza exige del profesorado una sólida y amplia formación en el campo disciplinario en el que se inscribe la asignatura que imparte.
Se identificaron seis artículos que evidencian una significación de la ingeniería sostenida en un énfasis tecnológico; en esta significación, diseño e innovación se consideran elementos nucleares de la ingeniería, vinculados estrechamente con el desarrollo tecnológico. Desde este marco significativo se asume que la tecnología es producto de la investigación aplicada y, por lo tanto, la formación del ingeniero debe responder a esa lógica de producción del conocimiento.

En esta significación se identifica un intento de responder a las condiciones político-económicas que configuran las actuales economías del conocimiento; éstas plantean demandas a las instituciones de educación superior tecnológica en el país relacionadas principalmente con la pertinencia de los programas y procesos formativos ante los entornos cambiantes y las nuevas lógicas de producción, difusión y consumo de conocimiento.

Aunque hay relaciones estrechas entre esta significación y la concepción operativainstrumental de la ingeniería, principalmente en su interés por responder a las exigencias del mercado laboral actual, se distingue en su manera de entender la relación ciencia-tecnología, ya que en esta significación se reconoce una imbricación entre ambas, producida en el marco de relaciones interdisciplinarias, en las que las racionalidades científica y técnica establecen algunos diálogos.

Por otro lado, la consideración de la habilitación para resolver las demandas del mercado laboral también conlleva efectos formativos diferenciados, en tanto en la visión operativa se busca responder a demandas específicas y concretas en un espacio laboral determinado, mientras en la visión tecnológica se reconocen contextos complejos y dinámicos que pueden plantear demandas diversas, ante las cuales el ingeniero debe responder integrando y movilizando conocimientos (de distinta procedencia disciplinaria), así como habilidades, capaci- 
dades y actitudes que le permitan comprender y resolver los problemas que se le presentan en los ámbitos profesionales-laborales en que se desarrolla.

\section{Significación socio-tecnocientífica}

La significación socio-tecnocientífica de la ingeniería parte de un cuestionamiento a las relaciones jerárquicas y lineales entre ciencia y tecnología, resultado del reconocimiento de múltiples y complejas relaciones, así como de las implicaciones sociales, políticas, culturales y económicas que producen. En ese reconocimiento se puede leer la incorporación de una racionalidad social, expresada en preocupaciones éticas que orientan el establecimiento de compromisos ante las problemáticas medioambientales, de género y de desarrollo económico regional, entre otras. Desde esta significación se asume que la formación del ingeniero debe incluir una visión orientada a la construcción de una nueva ciudadanía; en ese sentido, la significación socio-tecnocientífica se articula con la noción de formación como transformación subjetiva. Resulta relevante señalar que a partir del entendimiento socio-tecnocientífico de la ingeniería se propician relaciones transdisciplinarias que posibilitan la convergencia de distintos campos de conocimiento, articulados por intereses sociales, científicos y tecnológicos compartidos.

Los cuestionamientos y reconfiguraciones que se proponen desde esta significación se remontan a los albores del siglo pasado, cuando, ante los fracasos de la modernidad (sostenida en el supuesto de la ciencia objetiva - apolítica y neutra- como base del orden y progreso social) evidenciados en las guerras mundiales, el holocausto, la catástrofe ambiental y los peligros nucleares, algunas perspectivas epistemológicas cuestionaron de fondo las relaciones disciplinarias y los posicionamientos ético-políticos que sostienen la ciencia y la tecnología. En este contexto se pueden ubicar, por ejemplo, la teoría crítica en ciencias sociales y, más cercano al campo de la ingeniería, el movimiento CTS (ciencia, tecnología y sociedad), desde el cual se han propuesto alternativas para pensar la ciencia y la tecnología de manera contextualizada y como un proceso social (López, 1998).

A pesar de que hace más de medio siglo que estas discusiones se vienen produciendo, sólo en dos de los trabajos revisados se aprecian acercamientos difusos a este sentido de la ingeniería, manifestados en la preocupación por incorporar una racionalidad social en los procesos formativos de los/las ingenieros/as, entendidos como ciudadanos con una responsabilidad social ineludible que pueden cumplir a partir de su ejercicio profesional. Cabe mencionar que estos acercamientos parecen más bien intuitivos, es decir, no se aprecia una base epistemológica-teórica que los sostenga y direccione.

\section{Conclusiones}

Es pertinente recordar que, con base en los supuestos teóricos de los que partimos, los resultados que hemos reportado no constituyen verdades absolutas ni plantean significados permanentes; son significaciones históricas que se configuran en entramados discursivos complejos. Por ello, intentamos bosquejar algunos hilos que se han tejido en las tramas significativas sobre la formación de ingenieros para dar cuenta, en la medida de lo posible, de sus condiciones de producción y de los significantes encadenados metonímicamente en la construcción de sentidos sobre ser y formar ingenieros en el TecNM, que enuncian los docentes en los foros de difusión y divulgación institucionales.

Entre estos hilos se pueden mencionar, sin agotar: la influencia de los organismos internacionales, los mandatos de la política públi$\mathrm{ca}$, las significaciones sedimentadas en torno al proceso formativo de ingenieros que subyacen en los modelos educativos, y las condiciones de producción tecnocientífica que se están 
generando en las sociedades actuales y que plantean nuevas demandas y retos a las instituciones de educación superior. Elementos heterogéneos y múltiples que se articulan en tejidos complejos e imprevistos, desde los cuales los docentes se posicionan para entender y actuar la formación de ingenieros. Comprender estos posicionamientos resultó revelador en tanto permitió identificar asociaciones sedimentadas que es importante cuestionar, por ejemplo, entre formación y capacitación, significantes que establecen una relación casi sinonímica en algunos tejidos discursivos, o el entendimiento lineal y subordinando de las relaciones entre ciencia y tecnología, que sigue presente de manera predominante en la mayoría de las significaciones sobre ser ingeniero, enunciadas por los docentes del TecNM y que, posiblemente, dejarán huella en sus estudiantes.

Estas significaciones sobre el proceso formativo de ingenieros se expresan en las prácticas docentes cotidianas, de ahí que no resulten extrañas las actuaciones restringidas que desconocen las relaciones conceptuales y curriculares, así como la tendencia a pensar el aprendizaje como memorización de fórmulas o procedimientos y las limitaciones didácticas-pedagógicas (y, sobre todo, la poca preocupación por éstas), producidas por una estructura discursiva que se articula a partir de una significación operativa-instrumental de la ingeniería y un entendimiento limitado de la formación como capacitación.

Sin embargo, aunque incipientes, también se identificaron matices e intersticios en los que los posicionamientos se amplían para dar cabida a significaciones que conciben nuevas relaciones y lógicas entre ciencia y tecnología; éstas parten de reconocer una racionalidad social como núcleo formativo del ingeniero, desde la cual la formación puede conducir a un proceso de transformación subjetiva mediante el cual el ingeniero adviene en sujeto social.

El reconocimiento de las condiciones políticas, históricas y contingentes que se juegan en la configuración de significaciones sobre ser ingeniero en el TecNM abre la puerta a la posibilidad de repensar las concepciones y prácticas que guían los procesos formativos en este sistema; esto conlleva la necesidad de reestructurar su modelo educativo-curricular centrado en la trasmisión-aplicación de conocimientos disciplinares, para posibilitar un modelo que enfatice en la producción transdisciplinaria de conocimientos y en su transferencia social (Didriksson, 2014). Todo ello desde una visión crítica y ética que permita a los ingenieros entender y responder a las condiciones de las sociedades actuales, marcadas por nuevas lógicas de intelección, producción e interacción social y tecnocientífica, para poder transformar la realidad que habitan.

\section{REFERENCIAS}

Buenfil Burgos, Rosa Nidia (2019), Ernesto Laclau y la investigación educativa en Latinoamérica. Implicaciones y apropiaciones del análisis político de discurso, Buenos Aires, CLACSO.

Delors, Jacques (1996), La educación encierra un tesoro. Informe a la UNESCO de la Comisión Internacional sobre la Educación para el Siglo XXI, Madrid, Santillana/UNESCO.

De Alba, Alicia (2002), Currículum universitario, académicos y futuro, México, Plaza y Valdés.

De Saussure, Ferdinand (2004[1945]), Curso de lingüística general, México, Gedisa.

DíAz-Barriga, Ángel (2006), “El enfoque de competencias en la educación: ¿una alternativa o un disfraz de cambio?", Perfiles Educativos, vol. 28, núm. 111, pp. 7-36.

DidRIKsson Takayanagui, Axel (2014), "La universidad en la sociedad del conocimiento: hacia un modelo de producción y transferencia de conocimientos y aprendizajes”, Avaliação: Revista da Avaliação da Educação Superior, vol. 19, núm. 3, pp. 549-559.

Estrada Soto, Eufrocina (2012), "El enfoque de competencias", PulsoTec, año 9, núm. 17, pp. 13-14. 
Flores Dávila, Raquel (2012), "La creatividad y el imaginario social”, PulsoTec, año 9, núm. 18, pp. $29-30$

Fuantos Tobías, Edgar-Iván (2018), “¿La formación profesional? Sí ¿Y qué con mi educación sentimental?", PulsoTec, año 15, vol. 1, pp. 69-71.

Gadamer, Hans-Georg (1998), Verdad y método, Salamanca, Editorial Sígueme.

García Calvillo, Olivia, Eugenia Rodríguez Contreras y Araceli-Elizabeth Rodríguez Contreras (2015), "La tecnología aplicada a la enseñanza de las ciencias básicas”, Anfei-Digital, núm. 2, en: https://www.anfei.mx/revista/index.php /revista/article/view/135 (consulta: 15 de noviembre de 2020).

Gobierno de México-SEP-Dirección General de Educación Superior Tecnológica (DGEST) (2012), Modelo educativo para el siglo XXI. Formación y desarrollo de competencias profesionales, México, DGEST.

Heidegger, Martin (1989[1927]), Ser y tiempo, México, Fondo de Cultura Económica.

JAkobson, Roman (1956), Fundamentos del lenguaje, Madrid, Ciencia Nueva.

Jiménez Ortiz, María del Carmen (2011), “El discurso mundial de modernización educativa: evaluación de la calidad y reforma de las universidades latinoamericanas", Espacio Abierto, vol. 20, núm. 2, pp. 219-238.

LACAN, Jacques (1984), El seminario libro 3. La psicosis 1955-1956, Buenos Aires, Paidós.

LACAN, Jacques (2009), "La instancia de la letra en el inconsciente o la razón desde Freud”, en Jacques Lacan, Escritos 1, México, Siglo XXI, pp. 460-495.

LAClaU, Ernesto (comp.) (1993), Nuevas reflexiones sobre la revolución de nuestro tiempo, Buenos Aires, Nueva Visión.

Laclau, Ernesto (2001), "Posición de sujeto, dislocación y falta”, en: https://studylib.es/ doc/4816634/2.-posici\%C3\%B3n-de-sujeto-dislocaci\%C3\%B3n-y-falta (consulta: 21 de septiembre de 2021).

LAClaU, Ernesto (2006), "Entrevista realizada por Alicia de Alba Ceballos", The Homestead, Evanston, Northwestern University, en: https://www.youtube.com/watch?v=D9 lmeUGykQA (consulta: 20 de septiembre de 2020).

Laclau, Ernesto y Chantal Mouffe (1987), Hegemonía y estrategia socialista, hacia una radicalización de la democracia, Buenos Aires, Fondo de Cultura Económica.

López Cerezo, José-Antonio (1998), “Ciencia, tecnología y sociedad: el estado de la cuestión en Europa y Estados Unidos", Revista Iberoamericana de Educación, vol. 18, pp. 41-68. DOI: https://doi.org/10.35362/rie1801091

López Martín, Ramón (2017), "Hacia una innovación docente de calidad en la educación superior. Claves para la reflexión", Foro Educacional, núm. 28, pp. 11-28. En: https://dialnet.unirioja.es/servlet/articulo?codigo $=6429501 \quad$ (consulta: 25 de noviembre de 2019).

Lyotard, Jean-Françoise (1983), Le différend, París, Editions de Minuit.

Maldonado Maldonado, Alma (2000), "Los organismos internacionales y la educación en México. El caso de la educación superior y el Banco Mundial", Perfiles Educativos, vol. 22, núm. 87 , pp. 51-75.

Moguel León, Óscar-Oel (2016), "La educación superior tecnológica y su desarrollo en la sociedad del conocimiento. El caso del Instituto Tecnológico de Comitán”, Revista Electrónica de Investigación e Innovación Educativa, vol. 1, núm. especial, pp. 30-38.

Morales, Magda (2019), "La formación de ingenieros en los modelos educativos del Tecnológico Nacional de México: un significante ausente”, Revista de la Educación Superior, vol. 48, núm. 192, pp. 141-164.

OECD (2019), Higher Education in Mexico: Labour market relevance and outcomes higher education, París, OECD Publishing.

Palacios Reyes, Cecilia Guadalupe y Alejandrina Dávila Esquivel (2013), "El modelo educativo con enfoque por competencias", PulsoTec, año 10, núm. 19, pp. 7-8.

Pineda Armendáriz, Elva-Rosaura (2014), "La ingeniería y los retos del 2021”, PulsoTec, año 11, núm. 21, pp. 3-4.

Poblano Ojinaga, Eduardo-Rafael (2012), "El QFD en el proceso educativo", PulsoTec, año 9, núm. 18 , pp. 15-19.

Poblano Ojinaga, Francisco-Agustín y Ana-Abigail Adame Gutiérrez (2016), "La evaluación: el eslabón perdido de la educación basada en competencias", PulsoTec, año 13, vol. 1, pp. 21-26.

Ríos Flores, Jorge-Antonio y Reinalda Soriano Peña (2017), “Competencias profesionales de los ingenieros industriales; el caso del Tecnológico Nacional de México, campus Saltillo", Pistas Educativas, vol. 38, núm. 124, pp. 236-258.

Romero Jiménez, Alejandro (2013), "Desempeño en la universidad, desempeño en el trabajo", PulsoTec, año 10, núm. 19, pp. 5-6.

Romero Jiménez, Alejandro (2014), "Educando a la generación del milenio", PulsoTec, año 11, núm. 21, pp. 9-11.

Romo Rivera, Ileana y Kira Zamora Amén (2013), “Seguimiento de egresados”, PulsoTec, año 10, núm. 19, pp. 15-16.

RorTy, Richard (1990), El giro lingüístico, Buenos Aires, Paidós.

SAucedo Robles, Óscar y José-Omar Saldívar Correa (2014), "Promoviendo el emprendimiento", PulsoTec, año 11, núm. 21, pp. 11-12.

SAUR, Daniel Guillermo (2005), "Adiós a Derrida", en Ana María Martínez de la Escalera (ed), Adiós a Derrida, México, UNAM-Instituto de Investigaciones Filológicas, pp. 101-105. 
Serrano Salas, María de Jesús y Javier Castillo Muro (2014), "Atributos para el diseño e innovación”, PulsoTec, año 11, núm. 21, pp. 21-22.

Tecnológico Nacional de México (TecNM) (2015), Modelo de educación dual para el nivel licenciatura del Tecnológico Nacional de México, México, TecNM.

Urbina Amador, Ana-Lilia (2011), "Competencias en la educación superior", PulsoTec, año 8, núm. 16, pp. 13-14.

VÁzquez Peña, Diana-Margarita y Saúl Martínez Fonseca (2018), "Experiencias de formación dual en el Instituto Tecnológico de La Laguna", PulsoTec, año 15, vol. 1, pp. 21-30.
Velasco Aguilar, Aletia-Imperia, Heber-Abiu Hernández González, Rosy-Ilda Basave Torres, Santiago Erwin Beutelspacher, María-Candelaria Gutiérrez Gómez e Imelda Valles López (2019), "Herramienta m-learning en el caso de nivelación en lógica y fundamentos de programación”, Revista Tecnología Digital, vol. 9, núm. 1, pp. 29-36.

Weiss, Eduardo y Enrique Bernal (2013), "Un diálogo con la historia de la educación técnica en México", Perfiles Educativos, vol. 35, núm. 139, pp. 151-170. 